\title{
Toward a Human Vaccines Project
}

\author{
Wayne C Koff, Ian D Gust \& Stanley A Plotkin \\ Technological advances in antigen discovery, genomics and immunological monitoring offer tremendous potential for \\ revolutionizing vaccine development. On 5-6 February 2014, 35 leading vaccine scientists met to consider how best \\ to harness these advances and spur innovation.
}

$V_{\mathrm{in}}^{\mathrm{a}}$ accines are among the most effective interventions in the history of public health, having led to the eradication of smallpox, the near eradication of polio and the prevention of substantial morbidity and mortality from infectious diseases ${ }^{1}$. Today, a large number of vaccines have been licensed for the prevention of bacterial and viral diseases, including virus-induced liver cancers and cervical cancers caused by hepatitis B virus and human papilloma virus, respectively ${ }^{2}$. However, for several diseases for which vaccines are urgently needed, strategies that have proven successful in the past are unlikely to succeed. This is due to vaccinology challenges common to various pathogens in addition to pathogen-specific issues. For example, genetic variation presents considerable challenges for the development of vaccines against influenza virus, human immunodeficiency virus (HIV), hepatitis C virus, blood-stage malaria and other pathogens and diseases ${ }^{3}$. For HIV and other antigenically variable viruses, the immunization strategies required for driving somatic hypermutation and affinity maturation of antibodies remain unclear. Defects in short-term effector memory responses may impede the ability to generate long-lived protective immune responses, which affects the efficacy of vaccines against pertussis and mumps in some populations and impedes the development of vaccines against other diseases ${ }^{4}$. Moreover, immunization strategies

Wayne C. Koff is with the International AIDS Vaccine Initiative, New York, New York, USA. lan D. Gust is in the Department of Microbiology and Immunology, University of Melbourne, Parkville, Australia. Stanley

A. Plotkin is with the University of Pennsylvania,

Philadelphia, Pennsylvania, USA, and Vaxconsult, Doylestown, Pennsylvania, USA.

e-mail: wkoff@iavi.org for generating specific, potent and durable protective immune responses have not been defined for several pathogens, including respiratory syncytial virus, the leading cause of respiratory infection in infants, as well as for dengue fever, a widespread mosquitoborne disease ${ }^{5}$.

Population-specific challenges also limit the efficacy of some licensed vaccines and pose hurdles for new vaccine development. For example, immaturity of the immune system may have a role in limiting vaccine efficacy in neonates, and senescence of the immune system causes similar challenges for the elderly ${ }^{6,7}$. Vaccines against rotavirus are more effective in the developed world than in developing countries, where interference from maternal antibodies and concomitant infections probably affect vaccine efficacy ${ }^{8}$.

Most vaccines now available confer protection via antibodies, and this can often be defined as a correlate of protection on the basis of in vitro assays. In contrast, there are many diseases in which $\mathrm{T}$ cell immunity may also be required, such as malaria, tuberculosis and cancer. Indeed, for such diseases, the lack of understanding of correlates of protection and the inability to induce robust and sustained effector and memory $\mathrm{T}$ cell responses with the present vaccine approaches are substantial challenges. Moreover, the identification of which of the many potential antigens are required for protection also impedes the development of vaccines against these diseases $^{9-11}$. These challenges and the limitations of animal models that can be used to predict vaccine-induced immune responses and vaccine efficacy in humans have suggested the need for a new, human immunology-based clinical-research initiative to accelerate the development of new and improved vaccines ${ }^{12}$.

\section{Technological advances}

Technological advances in antigen discovery, genomics and immunological monitoring offer the potential to revolutionize vaccine development by providing solutions to the challenges noted above. Reverse vaccinology, enabled by the sequencing of the genomes of relevant pathogens, as well as the identification and expression of large numbers of protein antigens and the screening of such antigens, have already led to the development and licensure of a vaccine against meningococcus type $\mathrm{B}^{13}$. Advances in the identification of neutralizing monoclonal antibodies and their pathways, computational biology and structural biology have also led to the development of two novel strategies for the design of vaccines to prevent the disease caused by respiratory syncytial virus in neonates and have re-energized efforts toward the development of vaccines against HIV and influenza virus ${ }^{14-18}$. Improved methods of synthetic biology have resulted in advances in the development of vaccines containing nucleic acids, including alphavirus replicon-amplified RNA-based vaccines against influenza virus ${ }^{19}$. Replication-competent and replication-defective virus vectors, which mimic the attributes of live attenuated vaccines, are showing promise for the development of viral vaccines against dengue, HIV and cytomegalovirus ${ }^{20,21}$. In addition, mass-spectrometry methods as sensitive as the analysis of high-avidity T lymphocytes for the detection of epitopes on cells modified by viruses, bacteria or tumor transformation are now becoming available to investigate targeted complexes of peptide bound to major histocompatibility complex class I, by probabilistic measures ${ }^{22}$.

Systems-biology approaches that identify innate signatures shortly after immunization have been used to predict the immunogenicity of the vaccine against yellow fever in humans ${ }^{23}$. 


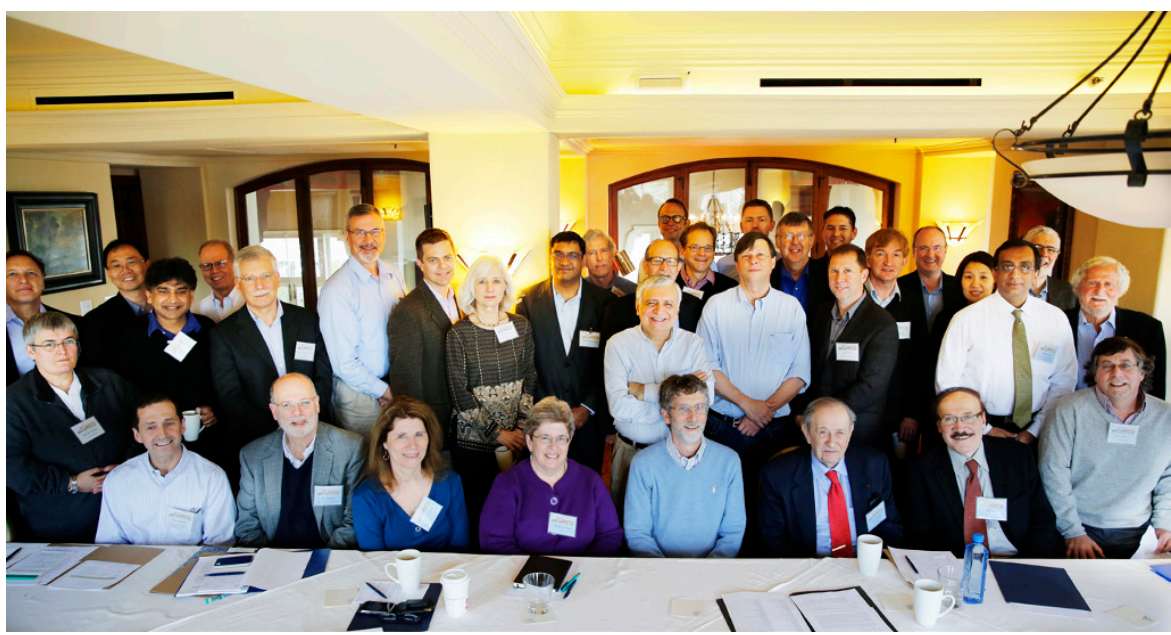

Figure 1 Participants in the Human Vaccines Project workshop held in La Jolla, California, on 5-6 February 2014.

Similarly, activation of the nutrition-sensing mechanism by a vaccine involving GCN2, a sensor of amino acid deficiency, correlates with the magnitude of the subsequent $\mathrm{CD} 8^{+}$ $\mathrm{T}$ cell response ${ }^{24}$. Thus, the ability to predict the immunogenicity and efficacy of a vaccine by innate signatures may offer great opportunities for streamlining future clinical development ${ }^{25}$. Next-generation sequencing and bioinformatics have already enabled probing of the antibody repertoire and greater understanding of the evolution of broadly neutralizing antibodies $^{26-28}$. In addition, the technological development of nanotechnologies and single-cell mass spectroscopy have now transformed immunological monitoring, enabling comprehensive investigation of cellular immune responses and opportunities for more effective probing of tissue-specific immune responses ${ }^{29}$. Collectively, building on advances in understanding of the integration of innate and adaptive immune responses, novel adjuvants and antigenpresentation strategies, as well as new insights into the development of human B cells, a new era in vaccine design is now emerging.

\section{Background and issues to address}

A human immunology-based clinical research initiative called the 'Human Vaccines Project' has been proposed to overcome barriers to accelerate the development of vaccines against major global diseases in humans ${ }^{12}$. To explore this concept in greater depth, 35 experts from industry, academia, government and nongovernmental organizations convened in La Jolla, California, on 5-6 February 2014 (Fig. 1). They were provided background information on key problems and unmet needs in vaccinology, strategies for designing next-generation vaccines, an overview of the proposed concept of the Human Vaccines Project and selected articles on vaccines against infectious diseases and cancers. The group was first asked to consider whether the proposed Human Vaccines Project was a viable concept that could provide substantial advances in the prevention and control of major diseases and, if so, to begin to develop the framework for a scientific plan for this project.

The group concluded that the concept for a Human Vaccines Project was meritorious, timely and potentially transformative and suggested that it focus on solving three major scientific problems that impede vaccine development to use this information to accelerate the development of new vaccines against major diseases. The group noted that to be successful, the project should ensure that its programs complement and augment existing efforts, be of sufficient scale to address the problems and foster innovation and technology development. Second, the project should create an enabling environment to facilitate small, hypothesis-driven clinical studies of humans. These efforts would include the cost-effective development and small-batch manufacture of immunogens, engaging regulatory agencies to ensure that such studies can be approved rapidly, and the maintenance of repositories of biological samples to facilitate the identification of novel biomarkers. Thus, outcomes should be achieved expediently. In the short and mid term, existing technologies and reagents should be used to generate meaningful outcomes. However, the project should also invest in innovation, technology development and the manufacture of immunogens and reagents to deliver solutions to key long-term problems. Fourth, the group recommended that the project focus its resources on vaccines against infectious diseases and cancer, while recognizing the potential translatable benefits of new knowledge toward the development of vaccines against allergies, autoimmune diseases and Alzheimer's disease. While the structure and governance of the Human Vaccines Project will be the subject of a future workshop, the group noted that accessibility and transparency of data would be critical. Finally, the group estimated that a minimum of US\$1 billion over a decade would be necessary for the Human Vaccines Project to achieve its objectives, and that close engagement with the vaccine industry and public-sector research agencies, along with academic centers and nongovernmental organizations, would be central to success.

\section{Problems and proposed solutions}

The group concluded that three major and related scientific problems impede the development of new and improved vaccines against infectious diseases and cancer. The most important problem cited was inadequate understanding of how to generate specific, potent, broad and durable immune responses in humans. Solving this problem would include gaining a more comprehensive understanding of why vaccine-induced immunity wanes over time; the cellular and molecular basis of immunodominance; the mechanisms that underpin adjuvant activity; the innate signatures that can be used to predict vaccineinduced immunogenicity and efficacy; and the signatures that can be used to predict the 'reactogenicity' or safety of a vaccine. Second, the group cited the lack of understanding of the precise antigens needed to elicit protective immunity against intractable pathogens and cancer as a major and solvable problem that is impeding next-generation vaccine development. Finally, the group noted that it is critical to understand how best to optimize vaccine efficacy in heterogeneous populations, including newborns, the elderly and those in the developing world, where future vaccines would be deployed.

The group also concluded that the most efficient and effective way to solve the problems noted above would be through a comprehensive assessment of human immune responses to licensed and experimental vaccines in rapid, focused and iterative clinical research trials. Thus, the creation of an environment that facilitates such trials and implementing these trials with unprecedented speed and scale would be the main strategic initiative of the Human Vaccines Project. Initial studies would be undertaken with immunogens already developed via partnerships with industry, vaccineproduct-development partnerships and other groups, while later studies would use immunogens commissioned specifically by the project. The group also noted that close integration of 
the project with ongoing product-development programs for disease-specific targets would be essential for achievement of the project's mission to accelerate the development of vaccines against infectious diseases and cancer in humans.

A big-science initiative that could facilitate the project's success would be delineation of the human 'immunome' (all genes and proteins associated with the immune system). The human adaptive immune system is able to generate an enormous diversity of B cell and $\mathrm{T}$ cell antigen receptors that facilitate molecular recognition of foreign pathogens and host molecules. The diversity of antibody-encoding genes in the naive repertoire (before somatic mutation driven by encounter with antigen) is estimated to be on the order of $\sim 1 \times 10^{11}$ sequences, and the diversity of $\mathrm{T}$ cell antigen receptors may be even larger. In the past, such a population size seemed unapproachable. However, with the increased efficiency of gene-sequencing technologies, bioinformatics and innovations in the management of large data sets, it now appears feasible to determine the sequence of all immunoreceptors in the naive cell compartment of the human adaptive immune system. Such a 'human immunome' database would provide new and additional tools to scientists for the substantial improvement of vaccine design.

Similarly, building from the 'human immunome' initiative and the series of iterative clinical research trials, the group proposed that large-scale antigen-discovery efforts aimed at identifying antigens required for inclusion in vaccines would initially use antigen-discovery technologies now available and could be enhanced by further investment in technology development. Finally, to address the problem of variable vaccine efficacy, the group envisioned comparative clinical research trials of heterogeneous populations and studies aimed at elucidating the effect of the human microbiome and other factors on vaccine efficacy. While emphasizing human clinical research trials as the core strategy for addressing these problems, the group noted that once sufficient data emerge from such trials, it will be important to compare their results with those obtained from animal models in an effort to determine whether any preclinical model(s) predict human vaccine immunogenicity and efficacy. The Human Vaccines Project is envisioned to accelerate next-generation vaccine development by various strategies (Fig. 2).

\section{Conclusions and next steps}

Vaccine development is among the greatest successes of modern medicine, yet strategies that have worked in the past are proving inef- fective for a range of important infectious diseases and cancers. For such diseases, vaccinology challenges common to multiple diseases and pathogen-specific issues pose major obstacles. The limitations of using animal models to predict human immune responses and technological advances in antigen discovery and immunological monitoring have led to a proposal for a large-scale Human Vaccines Project to systematically address these problems. The proposed project's working group identified major scientific problems that are impeding vaccine development and considered approaches for solving such problems. It also suggested a focus on selected infectious diseases and cancer and highlighted minimum resource requirements. The group also noted the importance and increasing interest of industry in working with academic partners as a critical success factor for a Human Vaccines Project. The group concluded that comprehensive assessment of human immune responses to licensed and experimental vaccines in rapid, focused and iterative clinical research trials could create novel strategies for the generation of long-lived protective immune responses in humans and thus enable the accelerated development of vaccines against infectious diseases and cancer.

The next steps toward the implementation of a Human Vaccines Project will be to gain further input from the project's steering committee and other key stakeholders on scientific priorities and resource considerations that emerged from this workshop, which will lead to further development and refinement of the project's scientific plan. Those activities will then be followed by consultations focused on potential options for the organizational structure, leadership and governance of the project, culminating in the development of the project's business plan. Finally, consultations will be held with key global stakeholders from industry, academia, government and nongovernmental organizations to gain consensus on the project's scientific and business plans, the identification of initial funding sources and short-term milestones. Just as the Human

\section{Human vaccines project}

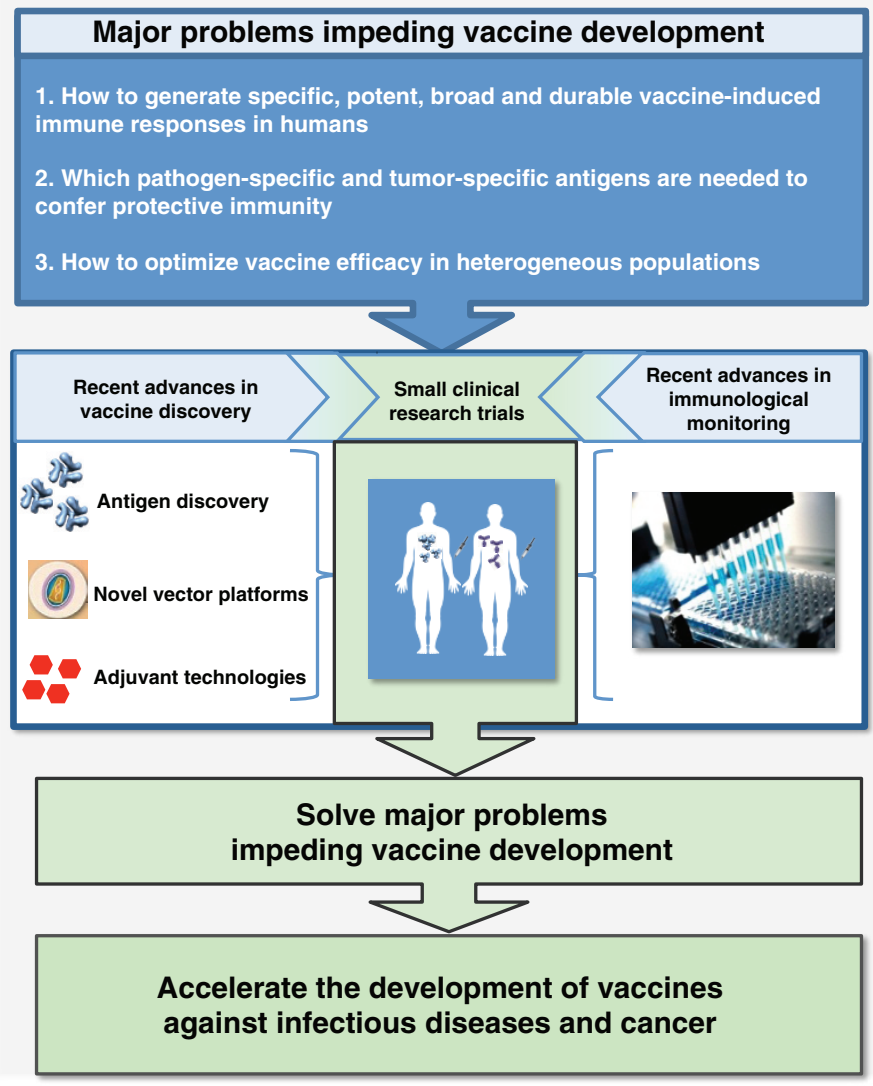

Figure 2 The Human Vaccines Project. Strategies for this project include identifying the major problems impeding vaccine development and implementing research and development programs, including small clinical research trials to solve the problems and thereby accelerate the development of new and improved vaccines against infectious diseases and cancer. Adapted with permission from ref. 12. 
Genome Project has transformed biomedical research and the future of drug development and personalized medicine, a Human Vaccines Project holds the promise of revolutionizing the development of vaccines against major diseases of the twenty-first century.

\section{ACKNOWLEDGMENTS}

We thank the Human Vaccines Project Working Group, which included Alan Aderem (Seattle Biomed), Rafi Ahmed (Emory Vaccine Center), Deborah Bae (Robert Wood Johnson Foundation), Ripley Ballou (GlaxoSmithKline), Maharaj K. Bhan (India Department of Biotechnology), Ashley Birkett (PATH Malaria Vaccine Initiative), Dennis Burton (The Scripps Research Institute), Danny Casimiro (Merck), Shane Crotty (La Jolla Institute of Allergy and Immunology), James Crowe (Vanderbilt University School of Medicine), Mark Davis (Stanford University School of Medicine), Hansi Dean (International AIDS Vaccine Initiative (IAVI)), Nathalie Garcon (GlaxoSmithKline), Ann Ginsberg (Aeras), Hana Golding (FDA), Rajat Goyal (IAVI), Barney Graham (National Institute of Allergy and Infectious Diseases (NIAID) Vaccine Research Center), Sanjay Gurunathan (Sanofi), Ian Gust (University of Melbourne), Barton F. Haynes (Duke Human Vaccine Institute, Duke University), Carole Heilman (Federal Liaison, NIAID), Adrian Hill (The Jenner Institute), Wayne Koff (IAVI), Antonio Lanzavecchia (Institute for Research in Biomedicine),
John Mascola (NIAID Vaccine Research Center), Julie McElrath (Fred Hutchinson Cancer Research Center), Douglas Nixon (George Washington University), Stanley Plotkin (Vaxconsult), Bali Pulendran (Emory Vaccine Center), Ellis Reinherz (Dana Farber Cancer Institute, Harvard University), Bill Schief (IAVI Neutralizing Antibody Center at The Scripps Research Institute), Robert Seder (NIAID Vaccine Research Center), Mark Slifka (Oregon Health and Science University) and Chris Wilson (Bill \& Melinda Gates Foundation); H. Dean, O. Shmaidenko and T. Schenkelberg for coordinating summary reports from group discussions in the workshop; L. Gieber for accessing 'pre-read' materials for the workshop; and S. Glass for logistics and organizational support for the workshop. Support for the Human Vaccines Project workshop was provided by a grant to IAVI (W.C.K.) from the Robert Wood Johnson Foundation.

\section{COMPETING FINANCIAL INTERESTS}

The authors declare no competing financial interests.

1. Centers for Disease Control and Prevention. MMWR Morb. Mortal. Wkly. Rep. 48, 621-629 (1999).

2. US Food and Drug Administration. Vaccines, Blood \& Biologics. http://www.fda.gov/BiologicsBloodVaccines/ Vaccines/ApprovedProducts/UCM093833 (2013).

3. Nabel, G.J. N. Engl. J. Med. 368, 551-560 (2013).

4. Wendelboe, A.M., Van Rie, A., Salmaso, S. \& Englund, J.A. Pediatr. Infect. Dis. J. 24, S58-S61 (2005).

5. Anderson, L.J. et al. Vaccine 31, B209-B215 (2013).

6. Lang, P.O. et al. Curr. Top. Med. Chem. 13, 2541-2550 (2013).
7. Wood, N. \& Siegrist, C.A. Curr. Opin. Infect. Dis, $\mathbf{2 4}$ 190-195 (2011).

8. Chan, J. et al. Vaccine 29, 1242-1247 (2011).

9. Greenwood, B.M. \& Targett, G.A. Clin. Microbiol. Infect. 17, 1600-1607 (2011).

10. Marinova, D., Gonzalo-Asensio, J., Aguilo, N. \& Martin, C. Expert Rev. Vaccines 12, 1431-1448 (2013).

11. Wenxue, M. Curr. Med. Chem. (5 February 2014).

12. Koff, W.C. et al. Science 340, 1232910 (2013).

13. Sette, A. \& Rappuoli, R. Immunity 33, 530-541 (2010).

14. Burton, D.R., Poignard, P., Stanfield, R.L. \& Wilson, I.A. Science 337, 183-186 (2012).

15. Correia, B.E. et al. Nature 507, 201-206 (2014).

16. Haynes, B.F., Kelsoe, G., Harrison, S.C. \& Kepler, T.B. Nat. Biotechnol. 30, 423-433 (2012).

17. Kwong, P.D., Mascola, J.R. \& Nabel, G.J. Nat. Rev. Immunol. 13, 693-701 (2013).

18. McLellan, J.S. et al. Science 342, 592-598 (2013).

19. Geall, A.J. et al. Proc. Natl. Acad. Sci. USA 109 14604-14609 (2012).

20. Hansen, S.G. et al. Nature 473, 523-527 (2011).

21. Parks, C.L., Picker, L.J. \& King, C.R. Curr Opin HIV AIDS 8, 401-410 (2013).

22. Reinhold, B., Keskin, D.B. \& Reinherz, E.L. Anal. Chem. 82, 9090-9099 (2010).

23. Querec, T.D. et al. Nat. Immunol. 10, 116-125 (2009)

24. Ravindran, R. et al. Science 343, 313-317 (2014).

55. Li, S. et al. Nat. Immunol. 15, 195-204 (2014).

26. Corti, D. \& Lanzavecchia, A. Annu. Rev. Immunol. 31, 705-742 (2013).

27. Liao, H.X. et al. Nature 496, 469-476 (2013).

28. Wu, X. et al. Science 333, 1593-1602 (2011).

29. Newell, E.W., Sigal, N., Bendall, S.C., Nolan, G.P. \& Davis, M.M. Immunity 36, 142-152 (2012). 